\title{
CONCEPTUAL DESIGN OF SMART FARMING SOLUTION FOR PRECISE AGRICULTURE
}

\author{
D. BUDAEV, A. LADA, E. SIMONOVA, P. SKOBELEV, V. TRAVIN, \\ O.YALOVENKO, G. VOSHCHUK \& A.ZHILYAEV \\ Knowledge Genesis Group, Smart Solutions, Samara State Technical University, Samara University, Moscow \\ Timiryazev Agricultural Academy, Peschanokopskaya Agro Group.
}

\begin{abstract}
The paper presents precise agriculture as a complex adaptive system with high level of uncertainty and dynamics, in which knowledge is forming experimentally and in a very enterprise-specific way. Is there any opportunity to learn the best practices from advanced precise farmers, transfer their knowledge and support everyday decision making for regular farmers? The concept of Smart Farming as an augmented $\mathrm{AI}$ solution for precise agriculture is proposed. The solution is designed as a digital eco-system (system of systems) of smart services, where each service, in its turn, is an autonomous AI system. The paper also discusses functionality of smart services for precise agriculture and the service-oriented architecture of the solution with $\mathrm{p} 2 \mathrm{p}$ interaction of services. Ontology-driven knowledge base and multi-agent technology are considered as the key technologies of the solution. The virtual "round table" for coordinated decision making of smart services is introduced. Finally, the paper presents results of the first applications, as well as the future steps and expected results.

Keywords: multi-agent technology, ontology, precise agriculture, real time, smart farming.
\end{abstract}

\section{INTRODUCTION}

The growing human population generates the increasing demand for more efficient food production under conditions of climate changes, constrained land resources, etc. One of the new perspective technologies is precise agriculture [1-3], which assumes ongoing monitoring of crops and fast precise reaction to unpredicted events in real time.

The quality and efficiency of precise agriculture can be significantly improved by using smart systems with the following capabilities for real-time decision making:

- real-time satellite- and drone-based pattern recognition of problematic situations with the use of hyper spectrum cameras (illness, insects, etc.);

- real-time event-driven adaptive resource management for machines, pesticides and other resources, including unmanned vehicles.

These opportunities could be designed and implemented as smart web-services available for farmers via constantly running Internet portal and mobile applications [4].

In this paper, we propose to design a new Smart Farming solution as a digital eco-system of smart services with the use of knowledge base and multi-agent technology. In the proposed concept, precise agriculture is considered as a complex adaptive system with high level of uncertainty and dynamics. The domain knowledge of precise agriculture for open-air farming is usually formed experimentally and in a very enterprise-specific way. It is not repeatable and not transferred from one enterprise to another. Is there any opportunity to learn the best practices from advanced precise farmers, formalize and transfer their knowledge and support everyday decision making for regular farmers?

The concept of Smart Farming as an augmented Artificial Intelligence (AI) solution for precise agriculture is considered. The solution is designed as a knowledge-based digital 
eco-system (system of systems) of smart services, where each service, in its turn, is considered as an autonomous AI system. The proposed concept will provide the opportunity to consider the decision-making process in agricultural enterprise as a negotiation process focused on solving conflicts and finding consensus among agents of experts in soil, wheat production, fertilizers, pesticides, finance, machines, etc.

Section 2 introduces the proposed concept and functionality of knowledge-based digital platform and eco-system for agriculture. The architecture of eco-system of smart services, as well as the virtual "round table" for coordinated decision making of agents representing smart services are presented in Section 3. Section 4 is focused on the first application and expected results. Section 5 presents the summary and outlines the next steps of developments.

\section{THE FUNCTIONALITY OF SMART SERVICES FOR PRECISE FARMERS}

According to Gartner's definition, "A digital ecosystem is an interdependent group of enterprises, people and/or things that share standardized digital platforms for a mutually beneficial purpose (such as commercial gain, innovation or common interest). Digital ecosystems enable you to interact with customers, partners, adjacent industries - even your competition" [5].

In our vision, a digital ecosystem is an open, knowledge-based, distributed and adaptive system of smart services ("system of systems"), which is able to self-organize and demonstrate other properties of complex systems [6]. As an example, let us consider how two key smart services could work autonomously and adaptively in strong cooperation:

- at any time smart pattern recognition can trigger re-scheduling of resources, for example, involving more fertilizers or pesticides;

- execution of plan may trigger new pattern recognition for control of results.

These two services can be considered as the "left" and "right" parts of agricultural "brain": one part is used for work with unstructured information, and the second part - for structural information, logical reasoning and calculations, helping farmers to reduce time-to-go.

In the proposed concept, smart services are considered as autonomous AI systems based on multi-agent technology - each service is represented by a software agent which is able to react to events, make decisions and coordinate them with other agents or humans [6]. The functionality of the solution includes the following agents of services (Fig. 1):

- Agents of Fields - multi-agent system for managing fields, including crop allocation, scheduling, optimizing and controlling;

- Agents of Monitoring - satellite / drone-based multi-agent system for recognizing patterns of illness, insects, etc.;

- Agents of Machines - multi-agent system for managing groups of machines for providing on-demand services for cultivating fields, bringing pesticides, etc.

- Agents of Pesticides - multi-agent system for bringing pesticides to agricultural fields;

- Agents of Fertilizers - multi-agent system for bringing fertilizers to fields;

- Agents of Finance - multi-agent system for finance planning, etc.

Agents of services can be implemented by the specific multi-agent system of low-level agents representing orders and resources, technology processes and tasks, products, spare parts and other entities of agricultural business. 


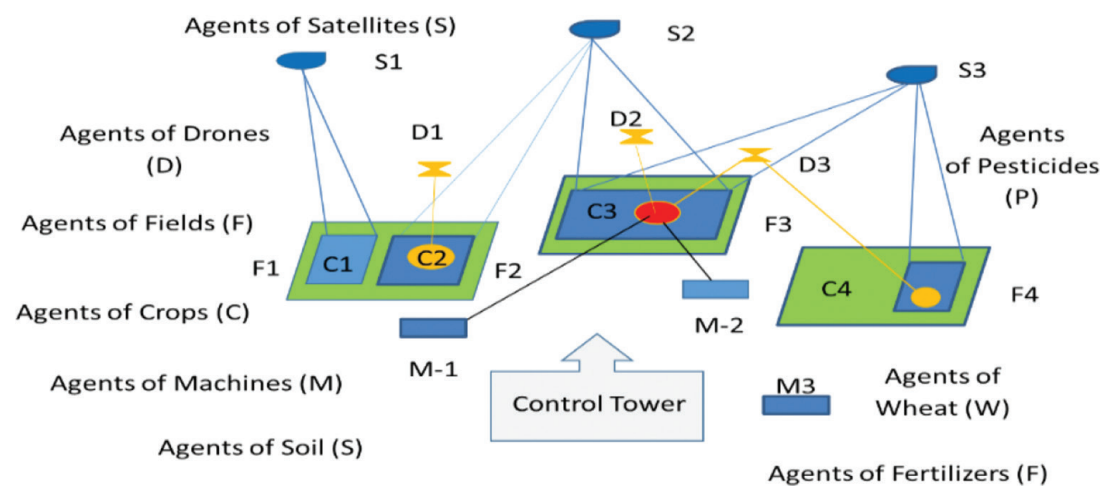

Figure 1: The multi-agent eco-system for supporting self-organization among services of satellites (S), drones (D), fields (F), machines (M) and others.

In the designed eco-system the images from satellites are continuously processed with calculation of NDVI indexes. They provide the global view on the fields, which helps recognize problematic areas for further, more detailed, investigation by swarms of drones or machines. When a problem pattern is recognized, the system recommends actions for solving the problem with the use of knowledge base, and makes adaptive re-scheduling of machines. Detected problematic zones require more attention from satellites and drones - until it becomes clear that the problem is solved.

\section{THE ARCHITECTURE OF THE SOLUTION}

The designed solution will be organized as service-oriented architecture with enterprise service bus and $\mathrm{p} 2 \mathrm{p}$ communication of smart services presented in Fig. 2.

The main part of the solution will be the knowledge base of precise farmers, which contains the following components:

- agricultural problem domain ontology represented by a semantic network of classes of concepts and relations with specific parts for wheat, pesticides, etc.;

- ontological model of enterprise and its current scene with the states of all objects and processes, which will contain specific instances of the above-mentioned classes, for example, information on history and state of fields, machines and other resources.

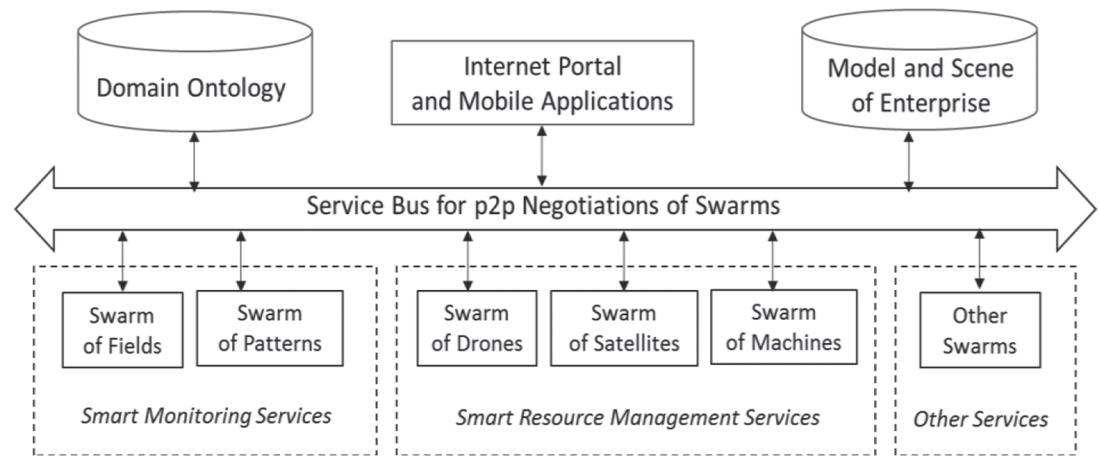

Figure 2: The architecture of Smart farming eco-system 
The architecture will be open for entering new smart services, which will register itself in the knowledge base with the use of yellow pages for subscriptions.

Each smart service is designed as an autonomous multi-agent system having its own event queue, virtual world of agents with plan of deliveries, UI components, database, decisionmaking log, etc. Smart services can generate orders and negotiate deals with other services and users in real time. For example, when a new user enters the system and provides information on his fields, the smart service of satellites can analyze its coordinates and compare them with routes for satellites - determining the place and time of receiving images and proposing its service to the user. Then it can also trigger the service of image processing and pattern recognition, drone scheduling, etc.

For coordinated decision making, special protocols are developed for agents of service negotiations, which support the "round table" negotiations among experts (Fig. 3).

During the "round table" negotiations, all agents follow their basic interests; however, when a conflict is detected, they use special techniques to define and solve the conflict.

Let us consider the example for the following team of agents:

- Agent of satellite is identifying a pattern of illness on one of the fields;

- Agent of drone plans detailed imaging;

- Agent of insects and Agent of illnesses are competing for the detected filed;

- Agent of agronomy schedules a visit to the field;

- Agent of agronomy recognizes that there is not enough fertilizers;

- Agent of fertilizer is scheduling extra amount of fertilizers to the field;

- Machines and humans are scheduled for the operation;

- Agent of field requests control of executed operation, etc.

Thus, the key feature of the solution is to demonstrate "team work" of agents with cooperative and adaptive behavior of services. Examples of screens for crop monitoring are presented in Fig. 4.

\section{Agent of field}

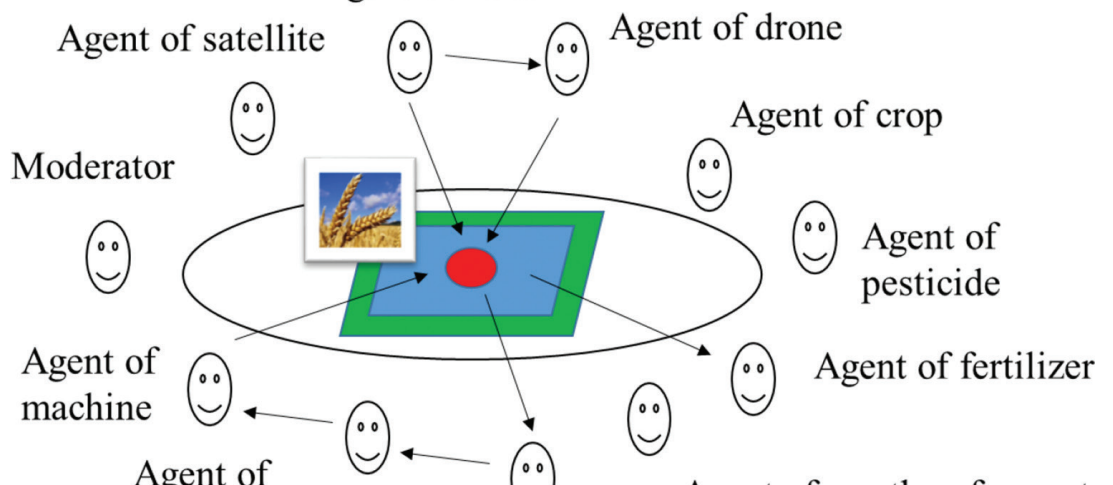

Agronomy

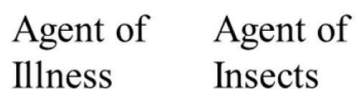

Figure 3: The Virtual Round Table for Coordinated Decision Making 


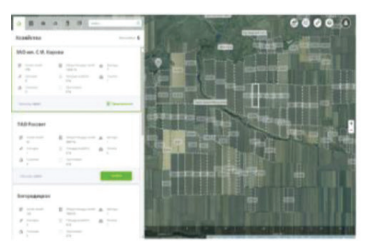

Switchover between farms

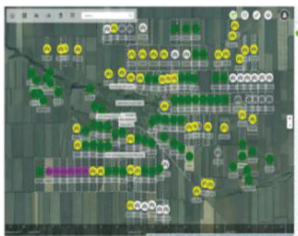

Crop rotation planning

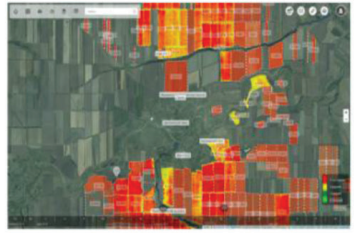

Fields map with the NDVI dato

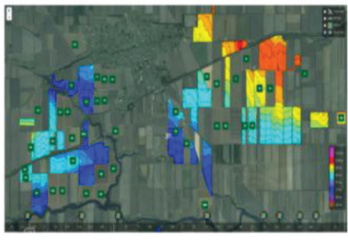

Example of maps with elevation contours on farm fields

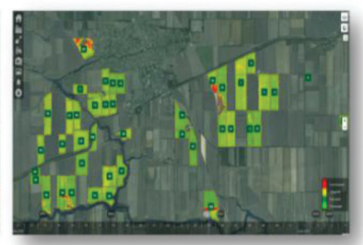

Changing the values of NDVI (July - September)

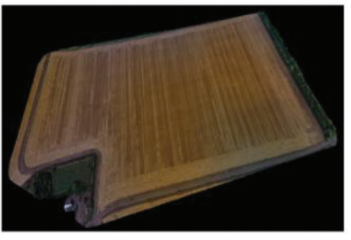

Example of 3D-madel of the field

Figure 4: The examples of solution screens

Another most important part of the solution architecture is domain ontology of agriculture and knowledge base for the enterprise. Ontology as a method and tool of Semantic web for specification of domain-specific knowledge is widely discussed in literature [7-8]. In the agriculture domain there is also a number of $\mathrm{R} \& \mathrm{D}$ activities focused on formalizing different aspects of plant genetics, biology, etc. [9-10].

In our developments, we are focusing on integrating "parts" of agricultural knowledge, which makes the technology of precise farming applicable for different regions and closely connected with economics. The content of the knowledge base includes the following: types of soils, sorts of wheat, classes of machines and mechanisms, illnesses of plants, insects, pesticides, fertilizers, etc. The first priority is given to the knowledge that is battle-proven in regional practice and forms a list of precedents and success stories.

Ontology Editor and examples of domain ontology and knowledge base of enterprise are presented in Figs 5 and 6.

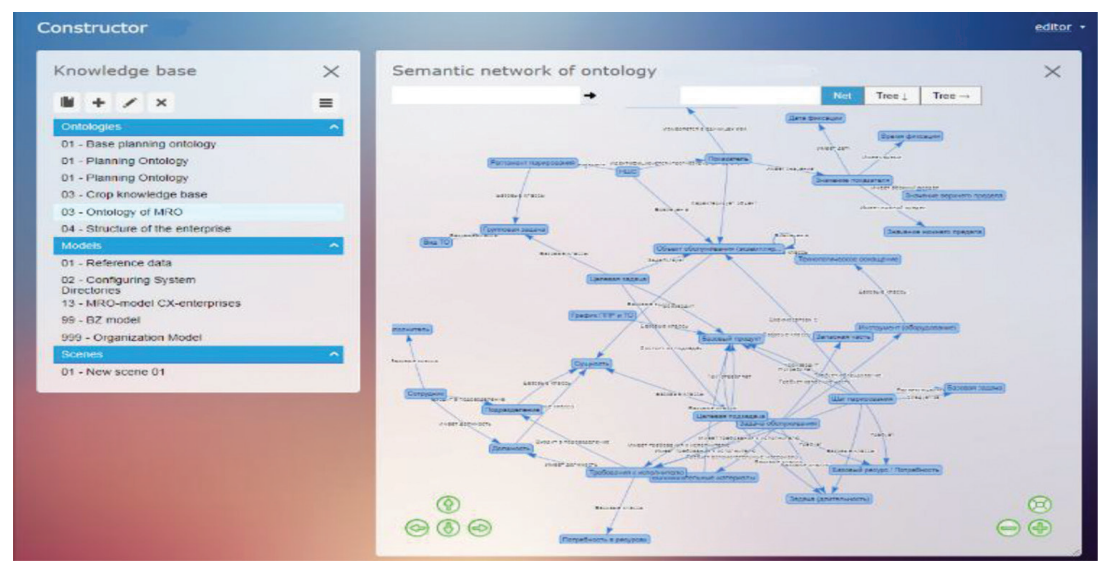

Figure 5: Ontology of agriculture 


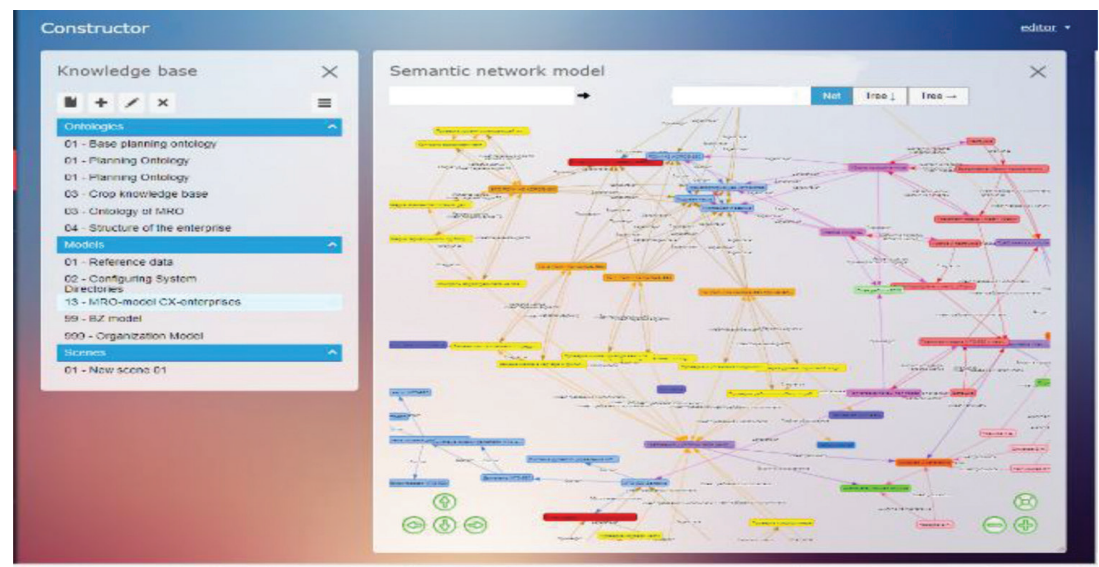

Figure 6: Ontological model of agricultural enterprise

The developed smart services for resource management are given in Figs 7 and 8.

The use of multi-agent technology together with ontologies makes it possible to allocate, schedule and optimize resources in real time taking into account the dynamics of events and situations that continuously change in practice.

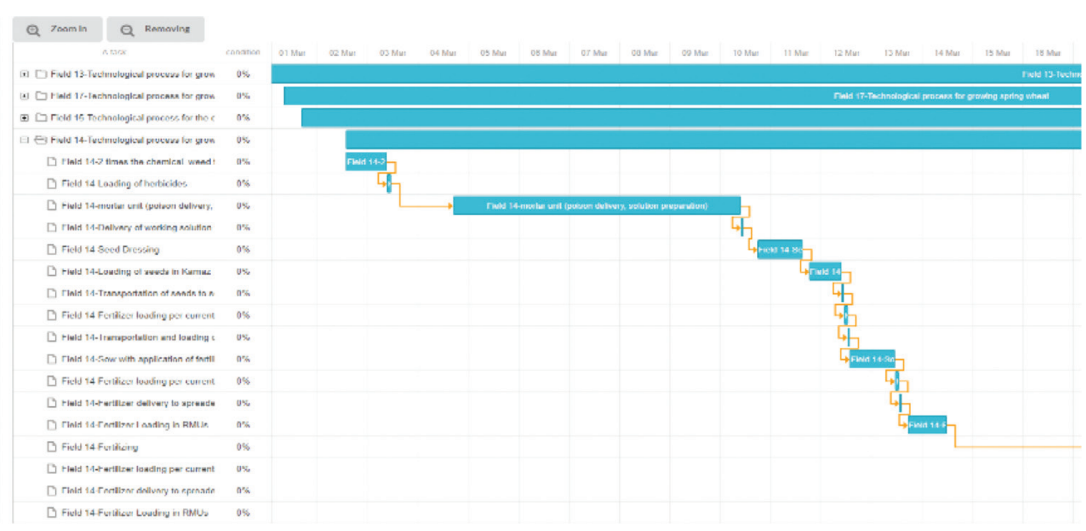

Figure 7: Example of machine operations scheduling

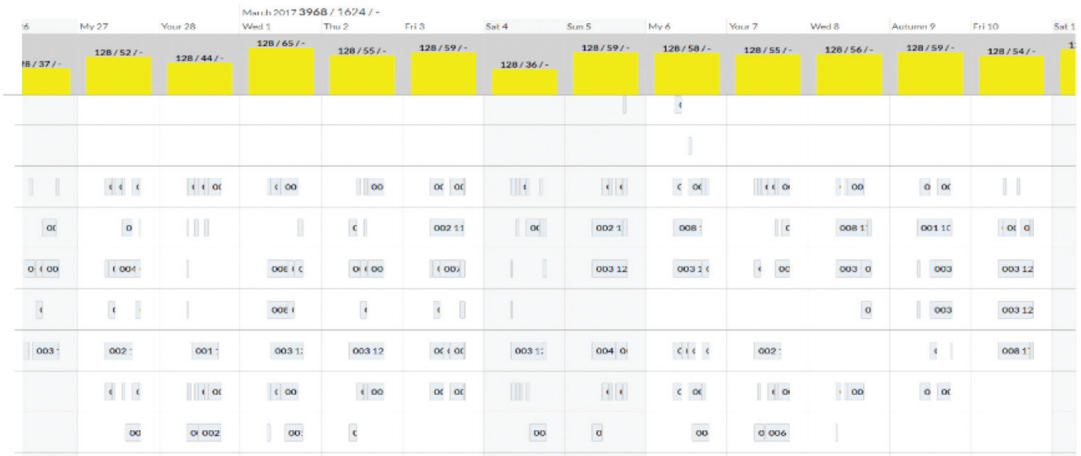

Figure 8: Dispatching daily operations 


\section{APPLICATION OF THE SMART FARMING SOLUTION}

The current stage of research is focused on conceptual design and modelling of key functional and architectural decisions.

Today the first prototype of the solution is designed and is currently under testing for Peschanokopskaya Agro Group in Rostov region, which has wheat fields with more than 30 000 ha of surface fragmented in smaller sections. Agronomy specialists need to monitor all these fields covering large distances on a daily basis (more than $700 \mathrm{~km}$ a day).

The system can target at least $5-10 \%$ increase of profitability for agricultural business as well as a number of other benefits for farmers:

- increasing crop productivity while improving crop quality by eliminating soil degradation through operational monitoring of fields;

- reducing harmful influence on the environment and the Earth's biosphere by using methods of precise agriculture based on calculation of exact fertilizers using operative information about vegetation indices;

- increasing the full transparency of business and payback using advanced IT-systems and decision support, etc.

In the future, the solution can be applied for a wide spectrum of agricultural enterprises, including manufacturing of wheat and rye, rice and soya fields, grape and apple gardens, coffee and tea plantations, olive groves and cork tree gardens, etc.

\section{CONCLUSIONS}

In this paper, we have introduced the concept of digital platform and eco-system for precise agriculture, which is related to the theory of complex adaptive systems.

In the proposed Smart Farming solution, decision-making support for farmers is based on self-organization of agents representing smart services for monitoring, resource planning, pesticides, fertilizers, etc. The architecture of the proposed solution is designed as a digital knowledge-based eco-system of smart services for precise agriculture, which uses enterprise service bus and $\mathrm{p} 2 \mathrm{p}$ communication of smart services. The virtual "round table" becomes the key mechanism for coordinated decision making of agents representing smart services. The use of multi-agent technology together with ontologies makes it possible to adaptively plan and optimize resources in real time, taking into account the uncertainty and dynamics of events.

The future works will be focused on developing ontology and knowledge base for precise agriculture of wheat and step-by-step implementation of smart services. It will also include detailed design of virtual "round table" protocols and implementation of autonomous smart services as well as methods and tools for learning from experience.

\section{ACKNOWLEDGEMENTS}

The work was supported by the Ministry of Education and Science of the Russian Federation in the framework of contract agreement No14.574.21.0183 - the unique identification number is RFMEFI57417X0183.

\section{REFERENCES}

[1] Sfiligo, E., Top 10 technologies in precision agriculture. September 9, 2016, available at https://www.therobotreport.com/top-10-technologies-in-precision-agriculture/ 
[2] Precise farming is an innovation in a system of resource-saving farming. The Federal Center for Agricultural Consulting and Retraining in the Agro-Industrial Complex, available at http://mcx-consult.ru/d/77622/d/tochnoe-zemledelie.pdf

[3] Nikolaev V.S. (ed.), Fundamentals of agricultural technology. Agriculture and plant growing [In Russian]. - Moscow: Bylina, 555 p, 2000, available at https://www.twirpx .com/file/1141268/

[4] Hopkinsm, M., 10 New Mobile Apps for Precision Agriculture, December 5, 2017, available at http://www.precisionag.com/service-providers/10-new-mobile-apps-forprecision-agriculture/?utm_source=PrecisionAg\&utm_medium=website \&utm_ campaign $=$ callout

[5] Digital Business Ecosystems \& The Platform Economy, available at https://www .gartner.com/technology/topics/business-ecosystems.jsp.

[6] Rzevski, G. \& Skobelev, P., Managing Complexity, WIT Press: Boston, 2014.

[7] Ontology Summit 2017 Communiqu'e - AI, Learning, Reasoning And Ontologies, available at https://s3.amazonaws.com/ontologforum/OntologySummit2017/Communique/OntologySummit2017Communique_v8.pdf

[8] Borgest, N.M. \& Korovin, M.D., Ontologies: current state, overview [In Russian]. Ontology of Designing, 2, pp. 49-55, 2013.

[9] Cooper, L., Meier, A., Laporte, M-A., Elser, J.A., Mungall, C., Sinn, B.T., Cavaliere, D., Carbon, S., Dunn, N.A. \& Smith, B., The Planteome database: an integrated resource for reference ontologies, plant genomics and phenomics. Nucleic Acids Research, 46(D1), pp. D1168-D1180, 2017. https://doi.org/10.1093/nar/gkx1152

[10] Repository for the Plant Ontology, available at https://github.com/Planteome/plantontology 